\title{
A SONIFICATION EXPERIENCE TO PORTRAY THE SOUNDS OF PORTUGUESE CONSUMPTION HABITS
}

\author{
Mariana Seiça, Pedro Martins, Licínio Roque and F. Amílcar Cardoso
}

\author{
CISUC, Department of Informatics Engineering \\ University of Coimbra \\ Pólo II - Pinhal de Marrocos \\ 3030-290 Coimbra, Portugal \\ \{marianac, pjmm, amilcar, lir\}@dei.uc.pt
}

\begin{abstract}
The stimuli for consumption is present in everyday life, where major retail companies play a role in providing a large range of products every single day. Using sonification techniques, we present a listening experiment of Portuguese consumption habits in the course of ten days, gathered from a Portuguese retail company. We focused on how to represent this time-series data as a musical piece that would engage the listener's attention and promote an active listening attitude, exploring the influence of aesthetics in the perception of auditory displays. Through a phenomenological approach, ten participants were interviewed to gather perceptions evoked by the piece, and how the consumption variations were understood. The tested composition revealed relevant associations about the data, with the consumption context indirectly present throughout the emerging themes: from the idea of everyday life, routine and consumption peaks to aesthetic aspects as the passage of time, frenzy and consumerism. Documentary, movie imagery and soundtrack were also perceived. Several musical aspects were also mentioned, as the constant, steady rhythm and the repetitive nature of the composition, and sensations such as pleasantness, satisfaction, annoyance, boredom and anxiety. These collected topics convey the incessant feeling and consumption needs which portray our present society, offering new paths for comprehending musical sound perception and consequent exploration.
\end{abstract}

\section{INTRODUCTION}

Auditory display has been writing its history as a scientific field for exploring sound as a medium of communication. In this journey, the main tendency in the research of auditory displays has been to explore its functional side and use sound to accurately represent data. However, a certain criticism marks this tendency, with many resulting experiences characterized as "unpleasant to listen to and difficult to interpret"[1], which hampers the potential of sonifications. Aesthetics has since risen as a potential concern for auditory communication [1][2], through which information can be understood in a designed context and engaging experience.

(c) (1) (9) This work is licensed under Creative Commons Attribution Non Commercial 4.0 International License. The full terms of the License are available at http://creativecommons.org/licenses/by-nc/4.0
A recent reflection about aesthetics focuses particularly on the subject perspective [2] and the role of the user and embodied cognition as a subject-position, with aesthetics becoming an essential dimension in sonification design and how auditory information is rendered and presented. Music is inevitably one form of organization; however, beyond a musical experience, sonification should be seen as an experiential, ecological or perception-action aesthetic [2], that the user actively listens to in search for information. As such, the aesthetic dimension is indispensable for the meaningmaking process, and cannot be separated from the informational / functional side. The aesthetics of sonification can thus have an essential role in auditory display communication, through which the information is encoded and musically structured by the system, and decoded by the user, exploring the aesthetics of experience, perception and the user as an embodied agent that can interact and explore the system. In this embodied setting, the concept of spatialization appears in creating a virtual or physical environment to explore the tridimensionality of sound [3], and for the user to actively explore while assimilating the auditory object. This exploration follows the phenomenological concept of apprehending a given phenomenon through a bodily experience, as "perception requires action" [4] and an active search in an environment where the body as a whole is inevitably embedded in that action. Our focus lies on the study and exploration of the role of the aesthetic dimension in the perception of auditory displays, studying how do people actively decode the auditory information, assimilating its context and iteratively apprehending its patterns and changes to decode its underlying information.

The first stage of our research focuses on the encoding stage, exploring how we can accurately represent a chosen dataset while composing a musical piece that provides an engaging, listening process, and how it is perceived by users as an auditory phenomenon that conveys information. For this experiment, we explored time-varying data, namely consumption data from SONAE, a Portuguese food retail company, and how its evolution through each day could be musically portrayed. The results, collected through a series of interviews to ten participants, showed some meaningful associations about the data, with emerging themes such as the idea of everyday life, routine, consumption peaks, and aesthetic aspects as the passage of time, frenzy and consumerism. The aesthetic perception also brought emerging sensations, from positive aspects of pleasantness and satisfaction, associated with the cohesiveness of the data representation day after day, to nega- 
tive sensations of annoyance, boredom or anxiety (among others), associated with the constant, steady rhythm and repetitive nature of the composition throughout the days.

The remainder of this paper is structured as follows. Section 2 comprises an overview of sonification projects using time-series, musical structures and embodied meaning-making processes. Section 3 discusses the data processing stage, and the filtering steps carried to gather the final dataset. Section 4 presents the musical mapping and its structure. Section 5 details the user testing conducted through a phenomenological approach, and the analysis of the results. The sixth and final section concludes the paper, listing the findings and future directions to take.

\section{RELATED WORK}

The concept of time-series with multiple variables is often represented through sonification, considered effective as an appropriate and successful tool to portray data evolution over time. The sound variations and regular patterns that we naturally perceive make most sound objects pertinent channels for conveying temporal dynamics and changes over time [2], and "tend to be more pleasant to the listener'[5].

Several works using time series served as examples from which to build this experiment. The first, called Quotidian Record and developed by Brian House in 2012, is a sound creation, engraved in a vinyl record, which translates his daily geographic routine for an entire year. Each place visited is harmonically mapped, translating not only its geographic coordinates (latitude and longitude), but also how he lives it and the time he spends there. Each rotation matches one day, composing a piece which brings out the underlying musical qualities of routine to create a portrait of a person's daily life [6]. The Climate Symphony [7] was created by Martin Quinn in 2011, where he used data from the chemical composition of an ice block in Greenland to translate into music the climatic changes endured by the great continental ice sheets. Living Symphonies [8] is a sound installation based on the fauna and flora of four ecosystems in the United Kingdom. The authors built a model that reflected the behavior, movement and daily patterns of every being in the wild, translating a network of interactions that formed the ecosystem.

Music is intrinsically connected to the study of auditory displays, serving many times as the main structure for the resulting auditory object. A sonification proposed by Dunn \& Clarke in 1999 is described as a collaboration that merges "scientific knowledge and artistic expression" [9], using information of the human DNA's coding units and possible combinations. The project resulted in the Life Music $C D$ [10] that achieves the exploration and refinement of a musical aesthetic to translate scientific data. This relationship meets a domain where ars informatica and ars musica merge together [11], and the goals of sonifications and musical pieces are blended. Klima-Anlage is "a walk-in sound installation" [12] that uses climate data from 1950, and a "global climate modeling experiment" that predicts climate variables evolution until 2100. It is an interactive installation, where the user can listen to the data from twelve regions, which includes precipitation data, wind data, radiation data and air temperature data, and also global greenhouse gas concentrations. Sound and video examples of several regions can be found in [13]. Another example is McGee and Roger's Musification of Seismic Data, who used straight audification processes to create musical compositions with a variety of timbres, through the "resampling, filtering, gran- ulation, timestretching, and pitch shifting" [14] of seismic vibrations, which resulted in compositions of the February 21st, 2011 Christchurch earthquake and of the 12th January, 2010 magnitude 7.0 Haiti earthquake [15].

Regarding the idea of embodiment and how cognitive processes are influenced by perceptual and bodily experiences, Roddy's research [16] focused on the aesthetic dimensions of sound in the process of meaning-making. To explore different embodied sonic dimensions and the embodied nature of auditory cognition, the author explored vocal gestures, environmental soundscapes and temporo-spatial motion, using human sounds, natural acoustic scenarios and temporal context. Examples for the first two experiments are available online [17], with data-driven compositions such as The Human Cost, Idle Hands and Doom \& Gloom which used statistical data of Irelands economic crash between 2007 and 2012.

\section{DATA PROCESSING}

The dataset used for this project was provided by the food retail company SONAE, as a result of a collaboration with our research group [5][18]. It gathers consumption data from 729 Portuguese supermarkets and hypermarkets of the food retail company SONAE, located throughout the country. The data was collected from the customers' loyalty cards, which can be used to accumulate several discounts and benefits. There are around 6 million active cards, shared by entire families for each household, which is a significant number for the ten million Portuguese population [5]. The data used for this experiment deals with transactions that occurred in 2014, from January 2nd to June 31st.

This dataset was well-fitted to what we intended to work upon, not only for its richness and size in terms of data variables and entries [5], but also for its pertinence in portraying everyday consumption habits and patterns of the Portuguese people.

Each transaction in the database corresponds to one single product, bought in a specific store, at a certain time, and with a particular cost. The products are also grouped within a product hierarchy of the company, with six levels [5]. The categorization that we used for this work was proposed by Maçãs, Martins and Machado [5], which aggregated the products into nine distinct categories: Grocery; Alcohol \& Sweets; Health care; Beauty; Clothes; Furniture; House Care; Culture \& Leisure and Pets \& Nature Care.

\subsection{Data Filtering}

The database has an average of 5 million rows of transactions for each day, which demanded several grouping and filtering steps to gather a manageable set of data to work upon. Having the spatialization stage and the user exploration possibilities still open for experimentation, there were two possible grouping forms for the data: by a spatial and temporal one. At the spatial level, the transactions could be grouped by district, county and store, allowing the user to explore different levels of depth by listening from the consumption sounds of an entire district to each store separately. The temporal level would focus on the pacing of the data timeline, grouping the transactions by a ten, thirty or sixty minute window for each ten seconds of the composition. This could allow the user to have an overall view of the six-month transactions in a few minutes, or to listen to just one day in the same amount of time.

To maintain a low level of complexity and number of files, we opted in this first stage to gather the data by spatial level, grouping 


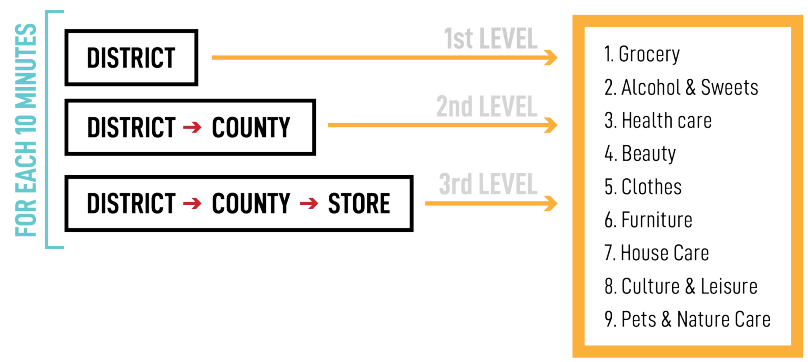

Figure 1: Data organization

the transactions first by category, and then by each 10 minutes for each district, county or store, preferable for the near-future spatialization experiences to explore different spatial depths (see Fig. 1). We also filtered the type of stores, keeping only the department stores of larger dimensions, namely the most known chain called "Continente". For this first experience, we retrieved the files of 12 days, from January 2nd of 2014 to January 12th for 22 districts (18 from Mainland Portugal, 1 from Madeira and 3 from Azores) in the three spatial levels, giving a total of 726 files to use.

\section{ORCHESTRAL MAPPING}

According to Vickers \& Hogg [11][19], sonification and music mutually imply each other in the design of sonifications, which can become, when focusing on the concepts of enjoyment and usefulness, "a mass medium for an audience with expectations of a functional and aesthetically satisfying experience" [1]. The link between science and art thus appear in uncovering the auditory reality of the dataset, surpassing the scientific method using the sound qualities, and specifically the natural "beauty and proportion in music" [20] to shape the listening experience not only for data communication, but even as a means for artistic expression. As such, an early decision made for this research was to devise this auditory experience through a musical form, which would translate the consumption narrative into a musical composition.

One of the main challenges while devising the mapping was how could we create a musical composition where the nine categories of products could be distinguished. Timbre emerged as the parameter with more perceivable variations that could be effective for discerning nine different variables, but even so the challenge of discerning nine instruments in a digital composition still remained.

The works of Grey [21] and McAdams et al. [22] regarding perceptual relationships between musical timbres are references in the study of timbrical similarities and common dimensions, using multidimensional scaling techniques to create spatial representations of timbres. Rentz [23] focused particularly in the perceptual discrimination of orchestral instrument families between musicians and non-musicians, with percussion and brass instruments sweeping the non-musicians attentions for longer, and strings capturing more the attention of musicians.

Thinking about acoustic communication brings the human into the center stage, and how can sound be understood from an human perspective. Truax's aspects [24] of (1). variety and richness of sounds, (2). complexity and the levels of information intended to communicate, (3). and a balance between the first two characteristics and the environment build the concept of an healthy soundscape. In this scenario, the sounds connect and merge with each other, the environment and the user, who is the main interpreter and focus of an acoustic communication.

We brought the notion of an orchestra as the main setup for our experience, with its forming sections naturally dividing the families of instruments, useful not only for organization purposes, but in this case as a method for grouping similar timbres which can be mapped to each category.

\begin{tabular}{|c|c|c|c|c|}
\hline \begin{tabular}{c|c|c|} 
PARAMETERS \\
EXPERIENCES
\end{tabular} & TIMBRE & PITCH & LOUDNESS & NOTE DENSITY \\
\hline 1 & Category & $\begin{array}{c}\text { Value of } \\
\text { Sales }(€)\end{array}$ & $\begin{array}{c}\text { Value of } \\
\text { Sales }(€)\end{array}$ & $\begin{array}{c}\text { Number of } \\
\text { Products }\end{array}$ \\
\hline 2 & Category & $\begin{array}{l}\text { Value of } \\
\text { Sales }(€)\end{array}$ & $\begin{array}{c}\text { Number of } \\
\text { Products }\end{array}$ & (Category) \\
\hline
\end{tabular}

Table 1: Implemented mapping

Using a parameter-mapping approach to sonification, we associated a set of musical parameters with the most relevant variables in the dataset (see Table 1). Two distinct mappings were made, along with a variation of the first with an added percussive element. The timbre, as the most variable and most naturally distinguishable parameter (we naturally recognize the difference between the sounds of two instruments) was mapped to the category types on both experiences, choosing nine different instruments to represent the nine categories (see Table 3). The instruments were chosen according to symphony orchestra sections (see Table 2), choosing a balanced set of timbres for each section to create an even-tempered sound. The sales value, which represents the amount of money each group of transactions cost, was directly mapped in both experiments to the pitch of the instruments. The pitch was adapted to each category, defining a range of values for each instrument to respect their natural range [25], and also for better distributing the sounds over lower and higher octaves which makes the instruments more distinguishable. To keep a consonant sound, a scale for which to choose the pitches was defined, namely the major (Ionian) scale, reusing part of the work developed in a previous sonification [26].

\begin{tabular}{|c|c|c|c|c|}
\hline WOODWINDS & BRASS & PERCUSSION & STRINGS & (KEYBOARDS) \\
\hline $\begin{array}{l}\text { Piccolo } \\
\text { Flutes } \\
\text { Oboes } \\
\text { English Horn } \\
\text { Clarinets } \\
\text { Bassons } \\
\text { Saxophones }\end{array}$ & $\begin{array}{l}\text { Horns } \\
\text { Trumpets } \\
\text { Trombones } \\
\text { Tubas }\end{array}$ & $\begin{array}{l}\text { Timpani } \\
\text { Xylophone } \\
\text { Marimba } \\
\text { Glockenspiel } \\
\text { Vibraphone } \\
\text { Chimes }\end{array}$ & $\begin{array}{l}\text { Harps } \\
\text { Violin } \\
\text { Viola } \\
\text { Cello } \\
\text { Double Bass } \\
\text { - } \\
\text { Guitar } \\
\text { Bass }\end{array}$ & $\begin{array}{l}\text { Piano } \\
\text { Pipe Organ } \\
\text { Harpsichord } \\
\text { Accordion }\end{array}$ \\
\hline
\end{tabular}

Table 2: Set of chosen instruments divided by orchestra sections

Loudness also changes moderately according to the sales value, within a range from the MIDI values of 77 , to maintain an audible minimum value, to the maximum of 127 , to emphasize the most sold categories. The choice to map both the frequency and loudness to the sales value, using different ranges of frequencies for each instrument, was made to emphasize the fluctuations between the values across the day, specially the hours with higher sales, and musically reinforce the data flow.

Besides the sales value, the dataset also gathered the number of products traded. As such, we can have a transaction with a high 
cost, but only for a single product, or a low-value purchase for a large amount of products. We chose to represent this variable through the note density of the melodies, directly mapping it to the rhythmic figures and their duration.

\begin{tabular}{l|l}
\multicolumn{1}{c|}{ Categories } & Instruments \\
\hline 1. Groceries & Grand Piano \\
\hline 2. Alcohol \& Sweets & Violin \\
\hdashline 3. Health Care & Vibraphone \\
\hline 4. Beauty & Flute \\
\hline 5. Clothes & Oboe \\
\hline 6. Furniture & Trombone \\
\hline 7. House Care & Bass \\
\hline 8. Culture \& Leisure & Guitar \\
\hline 9. Pets \& Nature Care & Timpani \\
\hline
\end{tabular}

Table 3: Association between the chosen timbres and the nine categories

In the first mapping, the instruments follow the same beat according to the initial BPMs, marking the tempo with whole, half, quarter or eighth notes (considering each 10 minutes a whole bar), or quarter, eighth, sixteenth and thirty-seconds notes, depending on the number of products. In the second mapping, each 10-minute beat is divided into nine beats representing the category order. For instance, the instrument that represents the first category plays in the first beat, the second in the second, and so forth. This "Arpeggio style", as we named it, does not represent the number of products through the note density, as it is constrained to the 1/9 beats in which each category plays, but through the loudness instead.

For this experiment, a simple interface was designed to browse through the possible days and districts, setting the deeper spatial levels aside to understand the overall patterns. Three software tools are used to produce the sonification: Processing, responsible for the dataset analysis, Max, to generate the musical composition through MIDI, and Ableton Live, to play the composition using VST's plugins. Audio and video examples of both experiments can be found online, of one weekday (January 2nd) and a weekend day (January 5th) for three districts (Lisbon the capital city, Coimbra as a medium-sized district with around 100.000 inhabitants, and Évora with around 50.000 inhabitants) ${ }^{1}$.

The process for perceiving the flow of this dataset was also a process of exploration: although we had a notion from a previous rhythmic sonification experience made with this dataset [5], this was also a learning process for us to uncover what we have, to understand how the sales for each place changes in each day, and how that can that change be musically portrayed.

\section{LISTENING EXPERIMENT}

As an "activity of perceptualization" [27], auditory displays demand a design process that understands how auditory information

\footnotetext{
${ }^{1}$ Dropbox Link - https://www.dropbox.com/sh/ $5 \mathrm{~s} 2 \mathrm{x} 7 \mathrm{vjmj}$ reuyfm/AAACxoZhRb $8 \mathrm{~b} 8 \mathrm{Hh} 86 \mathrm{zrv} 4 \mathrm{f} 8-\mathrm{a}$ ?dl $=0$
}

can be perceived by the user. With the goal of designing an auditory experience, it is fundamental to understand how the user positively engages with the artifact [28]. The users may know in advance that there is an underlying discovery process of the data to undertake, but the way they build that process and develop the auditory narrative depends on how they experience it, what they perceive, and the paths they choose to do it.

Phenomenology emerges as an approach to build this experience, where we can iteratively learn from the user's perspective how he/she perceives it to design a user-centred sonification exploration. Phenomenology, as "the study of experience from the perspective of the individual" [29], focuses on the experiential side of artifacts, and can be a powerful tool to gain insights into the user's actions and motivations, and how they shape their personal interactions with it.

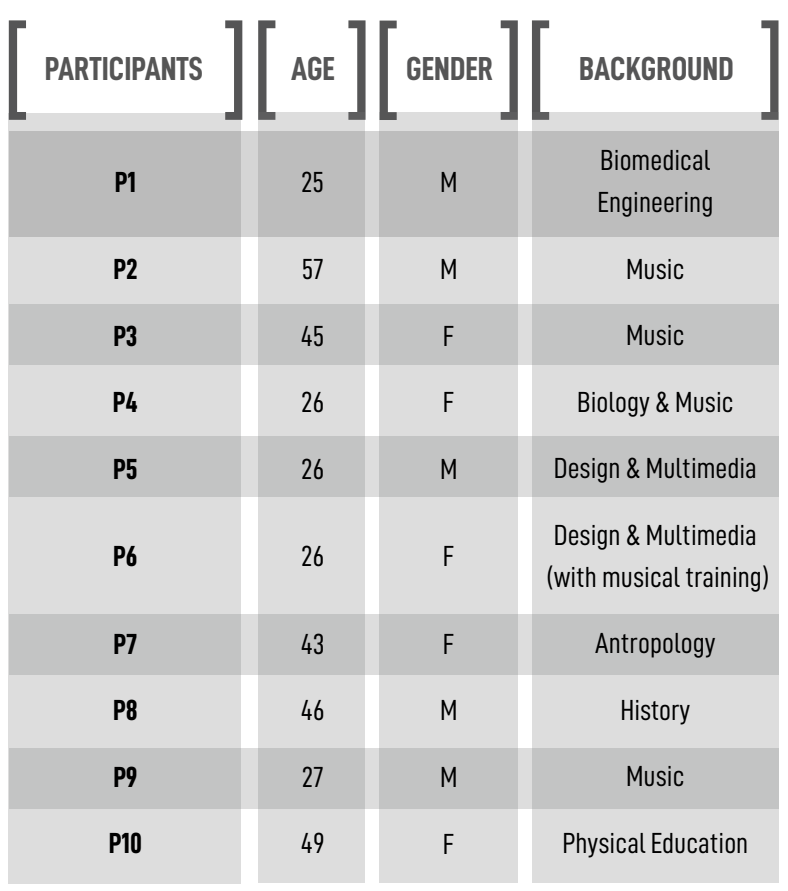

Table 4: Profiles of the participants for the evaluation

We decided to undertake a phenomenological approach to understand how the sonification is experienced as a phenomenon, uncovering meanings and common themes that the users associate while listening to it. It was not our goal with this type of evaluation to specifically detect if the exact mapping choices are understood, but instead what associations and sensations are perceived during the listening process, and how does each user perceives the sounds and its variations, and builds his/her acoustic narrative. This type of approach would allow us to observe the learning process of each listener, and consequently build our own discovery process of the dataset, creation methods, and its possible paths of exploration while shaping an aesthetic exploration.

\subsection{Methodology}

Ten participants were chosen to listen to the experience, and were submitted to an interview to document their perceptions. With 
$50 \%$ female and $50 \%$ male, and ages ranging from 23 to 57 years old, we tried to gather participants from different backgrounds (see Table 4), which would enhance the possibilities of a diversified set of perceptions. The interface was simplified for the evaluation as a continuous composition of the ten days (see Fig. 2), relative to the Coimbra district, which would allow a non-stop flow of the sonification to help building the learning process. A few commands were added, as the space bar to pause and play, providing moments of break in the music to focus on the interview exchanges, the possibility of skipping to the next day or return to the previous day with the left and right arrows, and the choice of changing the tempo (BPMs), from the default 100 BPMs, with the up and down arrows to experience a different scenario. A video example of the 10-day composition can be found online ${ }^{1}$.

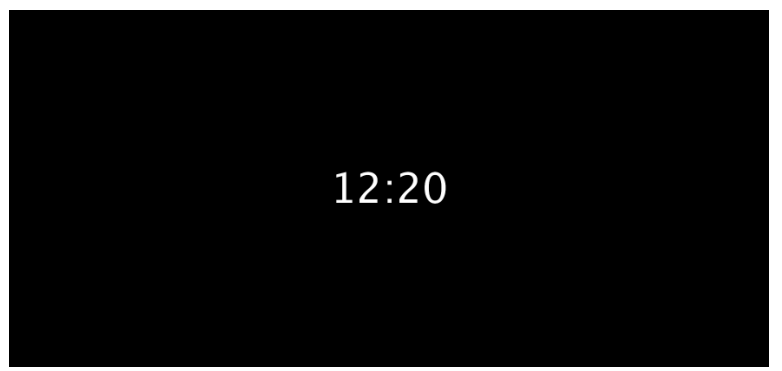

Figure 2: Screenshot of the interface created for testing

The interview was devised to create a receptive environment for the participants, and a flowing conversation with the interviewer to promote a safe, trustworthy setting to share their thoughts. In this scenario, we tried to ensure, as far as possible, a non-preconceived mindset from us, based on Husserl's epoché [30], which describes an attitude where interpretation is suspended to collect variations on the phenomena. Following the three initial hermeneutic rules [30], we presented the phenomenon in a clear, non-invasive way, diminishing the number of visual cues and other stimuli to understand what was immediate to the participants, and leading them to describe what they were experiencing without prejudgment from both parts. With this mindset, the interview was conducted according to the following steps:

1. It started with a minimal contextualization of the dataset, disclosing only that the composition represented consumption data from the Portuguese food retail company SONAE. The interface commands were also explained.

2. The descriptive, exploratory concept of the interview, with no right or wrong answers, was also emphasized, encouraging the participant to freely describe what associations and sensations arise during the listening process.

3. The first part of the interview was allocated for the user to freely navigate through the sound, changing or not the controllable parameters, describing to what concepts, scenarios or context the mind was travelling to, with or without relating to the consumption theme.

4. "Apprehending the Phenomenon" [31]: at a deeper level, the process of "seeking out structural or invariant features of the phenomena" [30] appeared in the next stage, refocusing the attention of each participant to the consumption context, and guiding him/her in apprehending the phenomenon [31] using his/her own metaphors and limits. In this stage, the intention was for the user to describe the connections he/she was making. We would ask the participant to clarify the emerging terms, sensations and how he/she was composing the narrative meaning, while we would hint, from time to time, new connections of those terms with sound changes and patterns he/she had previously mentioned. Hints would include: refocusing the attention to the numbers in the screen and what could they represent, to the variations of the instruments recognized or the musical characteristics perceived and their possible meaning. In this stage, new listenings were made to access the new paths that were suggested.

5. "Clarifying the Phenomenon" [31]: the use of imaginative variation was then included to propose new realities not perceived by the listener, creating new scenarios for reflection, how they were understood, and how could they change the intuitive settings. The clarification of the phenonemon [31] was therefore made with these new intended associations, which ended with the reveal of the mappings parameters to provide a complete scenario and a final discussion of the overall experience.

\subsection{Results}

After the interviews were conducted, the text was transcribed to identify a set of categories of interest which would provide the overall themes and interpretations perceived by the testers. Three categories were then labeled and distinguished (see Table 5): (1). the interpretations of the domain, to gather the scenarios and realities conveyed by the composition, (2). the overall sensations the sounds would provoke, (3). and the assessment of musical aesthetics, listing the musical parameters and correspondent variations the participants could distinguish. Within each category, we listed the key terms, and how they connected with each other and with parallel finding in other categories.

\subsubsection{Domain Perceptions}

Although the consumption context was given in the beginning of the interviews, the domain possibilities found surpassed it. The thematic of everyday life and daily routine appeared in $12 \mathrm{ci}$ tations of half of the participants, connecting the steady, regular rhythm to a normal day in a person's life. In this routine, words like habits, daily dynamics and automatism were mentioned, with one participant particularly describing a regular day where you "wake up, do your chores, reach noon and a peak of energy, take a nap, have a snack in the middle of the afternoon, and have a drink after dinner". It represents an energy variation, given by loudness variations and the rhythmic density. Participant 2 spoke about the "active day of a person, from 8:30am to $11 \mathrm{pm}$, that is our daily work flow, fast and tiring", joining the idea of movement and obligations to fulfill. The passage of time, hinted by the hours in the screen and the steady, repetitive tempo, is described as "the tick-tack of the clock", which conveys the feeling of construction, evolution, productivity, "that something is working and rising" and task assessment, "where we all work to reach the same goal, even doing slightly different things at different rates", with each instrument navigating in the given melodic scale.

The people's movement, the way they move across the day or inside the commercial area, was another of the most mentioned themes by four participants, with one comparing the piano from 


\begin{tabular}{|c|c|c|}
\hline $\begin{array}{c}\text { Domain } \\
\text { Interpretations }\end{array}$ & Occurences & $\begin{array}{l}\text { Number of } \\
\text { Participants }\end{array}$ \\
\hline Everyday Life & 12 & 5 \\
\hline Consumption Peaks & 11 & 7 \\
\hline People's Movement & 11 & 4 \\
\hline Consumerism & 10 & 4 \\
\hline Amount of People & 9 & 4 \\
\hline $\begin{array}{l}\text { Average } \\
\text { Consumption }\end{array}$ & 8 & 5 \\
\hline Passage of Time & 7 & 4 \\
\hline Frenzy & 5 & 4 \\
\hline Movie / Documentary & 5 & 3 \\
\hline Daily Search & 4 & 1 \\
\hline Trips & 2 & 2 \\
\hline Culture \& Leisure & 1 & 1 \\
\hline
\end{tabular}

\begin{tabular}{|c|c|c|}
\hline Sensations & Occurences & $\begin{array}{c}\text { Number of } \\
\text { Participants }\end{array}$ \\
\hline Annoyance & 5 & 4 \\
\hline Pleasantness & 4 & 2 \\
\hline Satisfaction & 4 & 2 \\
\hline Anxiety & 3 & 3 \\
\hline Lightness & 3 & 2 \\
\hline Fatigue & 2 & 1 \\
\hline $\begin{array}{l}\text { Aggressiveness I } \\
\text { Austerity }\end{array}$ & 2 & 1 \\
\hline Sadness & 1 & 1 \\
\hline Boredom & 1 & 1 \\
\hline Claustrophobia & 1 & 1 \\
\hline
\end{tabular}

\begin{tabular}{|l|l|l|}
\hline $\left.\begin{array}{l}\text { Assessment of } \\
\text { Musical Aesthetics }\end{array}\right]$ Occurences & $\begin{array}{c}\text { Number of } \\
\text { Participants }\end{array}$ \\
\hline High and Low Pitches & 17 & 6 \\
\hline $\begin{array}{l}\text { Steady Rhythm I } \\
\text { Regularity I } \\
\text { Time Measure }\end{array}$ & 16 & 7 \\
\hline Repetition & & \\
\hline Fast Rhythm & 8 & 5 \\
\hline Musical Composition & 5 & 4 \\
\hline Crescendo & 8 & 3 \\
\hline Layer Construction & 4 & 3 \\
\hline Melody & 3 & 2 \\
\hline Martial Style & 2 & 2 \\
\hline Volume / Loudness & 2 & 2 \\
\hline Mantra & 2 & 1 \\
\hline Instrument Distinction & 1 & 1 \\
\hline
\end{tabular}

Table 5: Analysis of the interviews, divided by three categories

$2 \mathrm{pm}$ to walking and two referring to the idea of paths, mazes, moving from aisle to aisle by the piano fast pace. The fourth participant even perceived the whole composition as a portrait of the consumers' rhythm, with the low tones representing the constant presence of people in the hypermarkets and the number of people, and the higher, faster tones the peak of people's access, or the ones that stay only for a short time. The focus on the people emerged as well through the link between the amount of people with the piano fast notes, the loudness variation and the rhythmic density, with the volume and the number of notes increasing in the peak hours of access and consumption. The context of consumption with consumption peaks emerged as such, perceiving "a stronger dynamic as higher consumption", the "hours across the day in which there are more purchases, especially in the evening", and "the rush hours of higher, frantic consumption", with two participants naming the difference between week and weekend days. The word frenzy is also mentioned by four participants to describe the shopping environment, particularly in the peak hours, relating to many people, agitation and even "the non-stop cash register hectically passing products person after person as an assembly line". The low, steady-paced sound was then curiously associated, by half the participants, with the regular amount of products bought, the standard, average of consumption, or to the essentials goods that people need to consume everyday, associated with the constant, low-pitched sound that seemed to mark a regular tempo.

Consumerism appears as an underlying subject, mentioned by one participant as an "unceasing daily quest" for what we need or think we need to buy, without calm and reflection. The impulsive attitude is pointed out by three participants, with the high, fast-paced piano representing "not daily products, but those who are highlighted" to gain the consumer's focus, where we think "I don't need it, but the price is nice", revealing a "certain urgency to buy, to consume".
Other surprising themes that emerged include thinking about the composition as a "soundtrack of an alternative movie", or a documentary or movie imagery, namely a "documentary about the human life, where they film mass people, mass consumption, mass movements in the search for progress", of "fast-paced life". Travelling was also mentioned, associating a "train station, the train dynamics, movement, leaving the monotony behind", and even an "intergalactic journey", associated with the symphonic, technological sound of the instruments.

\subsubsection{Sensations}

Regarding the sensations that arose from the listening experience, annoyance was mentioned by four participants, mainly associated with high-pitched sounds, which would become annoying and strident after a while. Nevertheless, it was characterized as pleasant and satisfying by four participants, which was associated by listeners as (1). being a "musification instead of sonification" (by a participant whose background is also working with data), (2). being "cohesive", despite its repetitive nature, that conveys the idea of people moving, (3). and providing an enjoyable moment, "that would be wonderful to be playing in a commercial area" and just feels like "going on a ride with no destination, alone, and embracing the moment through the music". At one point in the interview, the last participant that described this last scenario curiously asked if she had to be aware of more details, or if she could just "enjoy it", which can be a hint that the musical piece can provide an engaging listening process. Also in a positive spectrum, the composition was characterized by being light and soft, as if it represented "a pleasurable job".

In a negative spectrum, besides annoyance, it gave the idea of fatigue, "exhaustion after a day's work, and representing the tiring, repetitive movement of everyday duties". Sadness was also 
mentioned by a participant when played slowed (20 BPMs), and boredom as well, which was felt after a while associated with its repetitive nature. A sensation of anxiety was felt by three participants, which was associated by listeners to the fast rhythm and "a more intermittent, higher sound", which would convey the anxious, fast movement during peak consumption hours. One particular participant felt the steady, marked tempo as aggressive and "a bit dictatorial, almost as an imposing march", with another participant relating the march scenario to the work rhythm. An unnerving feeling of claustrophobia was particularly mentioned by one participant, again associated to the faster, high-pitched piano, recalling moments of being in a middle of a crowd, feeling muffled and thinking "I don't want to be here".

\subsubsection{Musical Assessment}

The assessment of musical aesthetics provided parameters already treated in relation with several domain interpretations and sensations, such as the acknowledgment of high and low pitches, a steady, regular rhythm, fast rhythm, repetition and loudness variations. Analyzing musical style, composition, and melody construction, was primarily done by the participants with a musical background, with four out of five naturally speaking about the role of each instrument in the composition, with "the winds setting the tempo and rhythm" and each instrument appearing successively as a new layer. It was also characterized as having an oriental, martial style, and even a mantra for having this repetitive, almost hypnotic nature. Multiple instruments were naturally distinguished, primarily the piano for every participant and winds for seven participants, with four or five participants with musical background distinguishing more than five instruments, when their attention was redirected to that task.

\section{CONCLUSION AND FUTURE WORK}

The focus of our investigation lies on the study of the influence of aesthetics in sound perception, primarily in data representation through auditory displays. We focused on how we could compose a musical piece that could be representative of consumption data from the Portuguese food retail company SONAE. Our goal was to consider the sonification experience as a phenomenon that the user perceives and assimilates, iteratively apprehending its patterns and changes to decode its underlying information. We applied a phenomenological approach to evaluate the experience, conducting a series of interviews to assess the users perception.

The evaluation carried out, with the goal of gathering the realities and associations sensed through the listening experience, provided some interesting results, with the consumption context indirectly present throughout the emerging themes. The idea of everyday life, routine, time passing over each day and how we move and carry on our day in a fast, frenetic rhythm are sensations intrinsically connected to the consumption context. The variations of the melody flow throughout a day of consumption are similar to the ones people live in their work day, with peaks of energy, peaks of responsibility, which repeat themselves day after day. Overall, the participants related the musical flow with a more human perspective of how we live each day nowadays in a fast, frenetic and stressful rhythm that dictates our modern routine. The evocation of a documentary or movie imagery about life in the twenty-first century, with mass people moving and working in search for progress, also translates this scenario.
Several suggestions and comments made by the participants defined an initial list of possible refinements and settings to experiment in the future. The amount of negative sensations, as annoyance, boredom or anxiety, could be reduced by exploring the effect of different tempo measures, and balance the differences between the high and low pitches, lowering the maximum range of pitch values to avoid the strident sensation, and lowering the intensity of low-pitched sounds, specially the trombone, which may be masking other timbres. The steady rhythm, with varied speed depending on the time of day, although it may help making the composition feel annoying or boring, it translates the expected repetitive nature of the data, and maybe why people felt the idea of routine and everyday life that is constant. This might be due to the categories with less variations, mapped coincidentally to low-pitched instruments (as the oboe and the trombone), which tend to mark the tempo and stand out. It should be noted that these are just speculative explanations, which demand further testings with a higher sample of interviewees to properly validate the mapping choices.

The emerging themes gave us a set of scenarios to further reflect upon, demanding new iterations on the mapping and how the different variables are balanced within the sound. This experience already demanded an active role of the user in a disembodied form. Further work might include interaction modes, which could provide a better support for active listening, namely a spatialized environment that could allow the user to move through the space, from store to store, and emphasize the idea of moving to search for information, to discover and internalize the auditory phenomenon. In this setting, we intend to explore the expected differences already noticed between stores, as the ones from cities of larger dimension and wider population have more variations across the day and higher levels of sales. We intend to explore how could these differences be perceived while exploring the auditory space, studying the concepts of interaction and perception aesthetics through this embodied process for user engagement.

\section{ACKNOWLEDGMENT}

Mariana Seiça is funded by Fundação para a Ciência e Tecnologia (FCT), Portugal, under the grant SFRH/BD/138285/2018. We would like to thank the retail company SONAE, and the members of the project Sonae Viz - Big Data Visualization for retail Catarina Maçãs and Evgheni Polisciuc for processing the data. We also thank the ten individuals for giving a part of their time in participating and contributing to this experiment.

\section{REFERENCES}

[1] S. Barrass and P. Vickers, The Sonification Handbook. Berlin, Germany: Logos Verlag, 2011, ch. Sonification Design and Aesthetics, pp. 145-172.

[2] P. Vickers, B. Hogg, and D. Worrall, Body, Sound and Space in Music and Beyond: Multimodal Explorations. London and New York: Routledge Taylor Francis Group, 2017, ch. Aesthetics of sonification: taking the subject-position, pp. 89-109.

[3] J. G. Neuhoff, The Sonification Handbook. Berlin, Germany: Logos Verlag, 2011, ch. Perception, Cognition and Action in Auditory Displays, pp. 63-86.

[4] D. Svanæs, "Interaction design for and with the lived body: Some implications of merleau-ponty's phenomenol- 
ogy," ACM Transactions on Computer-Human Interaction (TOCHI), vol. 20, no. 1, p. 8, 2013.

[5] C. Maçãs, P. Martins, and P. Machado, "Consumption as a rhythm: A multimodal experiment on the representation of time-series," in 2018 22nd International Conference Information Visualisation (IV). IEEE, 2018, pp. 504-509.

[6] B. House. (2012) Quotidian record. [Online]. Available: http://brianhouse.net/works/quotidianrecord/

[7] M. Quinn, "Research set to music: The climate symphony and other sonifications of ice core, radar, dna, seismic and solar wind data," in Proceedings of the 2001 International Conference on Auditory Display, Espoo, Finland.

[8] J. Bulley and D. Jones. (2014) Living symphonies. [Online]. Available: http://www.livingsymphonies.com/

[9] J. Dunn and M. A. Clark, "Life music: the sonification of proteins," Leonardo, vol. 32, no. 1, pp. 25-32, 1999.

[10] J. Dunn and M. A. Clarke. (1998) Life music. [Online]. Available: http://algoart.com/music.htm

[11] P. Vickers, "Sonification abstraite/sonification concrète: An 'aesthetic perspective space' for classifying auditory displays in the ars musica domain," vol. abs/1311.5426, 2013. [Online]. Available: http://arxiv.org/abs/1311.5426

[12] K. Groß-Vogt, T. Hermann, M. W. Jury, A. K. Steiner, and S. Kartadinata, Klima |Anlage-Performing Climate Data. Cham: Springer International Publishing, 2019, pp. 339355.

[13] K. Gro-Vogt, T. Hermann, M. W. Jury, A. K. Steiner, and S. Kartadinata. (2016) Klima |anlage: Perfoming climate data. [Online]. Available: http://klima-anlage.org

[14] R. McGee and D. Rogers, "Musification of seismic data." The 22nd International Conference on Auditory Display, Canberra, Austria, 2016.

[15] _ (2012) Haiti earthquake mw7.0 12th january *02010 - ryan mcgee. [Online]. Available: https://soundcloud.com/ seismicsounds/haiti-earthquake-12th-january

[16] S. Roddy, "Embodied sonification," Ph.D. dissertation, University of Dublin, Trinity College, 2015.

[17] - The human cost: Sonification and irelands economic crash. [Online]. Available: https://stephenroddy.bandcamp.com/album/ the-human-cost-sonification-and-irelands-economic-crash

[18] C. Maçãs, P. Cruz, P. Martins, and P. Machado, "Swarm systems in the visualization of consumption patterns," in 24th International Joint Conference on Artificial Intelligence, 2015.

[19] P. Vickers, The Routledge Companion to Sounding Art. Routledge, 2016, ch. Sonification and Music, Music and Sonification.

[20] S. Gresham-Lancaster, "Relationships of sonification to music and sound art," AI Soc., vol. 27, no. 2, pp. 207-212, May 2012 .

[21] J. M. Grey, "Multidimensional perceptual scaling of musical timbres," the Journal of the Acoustical Society of America, vol. 61, no. 5, pp. 1270-1277, 1977.
[22] S. McAdams, S. Winsberg, S. Donnadieu, G. De Soete, and J. Krimphoff, "Perceptual scaling of synthesized musical timbres: Common dimensions, specificities, and latent subject classes," Psychological research, vol. 58, no. 3, pp. 177-192, 1995.

[23] E. Rentz, 'Musicians' and nonmusicians' aural perception of orchestral instrument families," Journal of Research in Music Education, vol. 40, no. 3, pp. 185-192, 1992.

[24] B. Truax, Acoustic communication. Greenwood Publishing Group, 2001.

[25] Ranges of orchestral instruments. [Online]. Available: http://www.orchestralibrary.com/reftables/rang.html

[26] M. Seiça, R. B. Lopes, F. A. Cardoso, and P. Martins, "Sonifying twitter's emotions through music," in Lecture Notes in Computer Science, Music Technology with Swing, Revised Selected Papers of the 13th International Symposium on Computer Music Multidisciplinary Research, M. Aramaki, M. Davies, R. Kronland-Martinet, and S. Ystad, Eds. Berlin, Heidelberg: Springer, in press.

[27] B. N. Walker and G. Kramer, Ecological Psychoacoustics and Auditory Displays: Hearing, Grouping, and Meaning Making. New York: Elsevier Academic Press, 2004.

[28] Z. Bilda, Interacting: Art, Research and the Creative Practitioner. UK: Libri Publishing, 2011, ch. Designing for Audience Engagement, pp. 163-181.

[29] S. Lester, "An introduction to phenomenological research," 1999.

[30] D. Idhe, Experimental Phenomenology. New York, USA: State University of New York Press, 1977, ch. Phenomena and the Phenomenological Reductions, pp. 29-54.

[31] M. T. Bevan, "A method of phenomenological interviewing," Qualitative health research, vol. 24, no. 1, pp. 136-144, 2014. 